\title{
Article
}

\section{The Guangzhou Twin Eye Study: 2019 Update}

\author{
Xiaohu Ding ${ }^{1}$, Wei Wang ${ }^{1}$, Jane Scheetz ${ }^{2}$ and Mingguang $\mathrm{He}^{1,2,3}$ \\ ${ }^{1}$ State Key Laboratory of Ophthalmology, Zhongshan Ophthalmic Center, Sun Yat-sen University, Guangzhou, China, ${ }^{2}$ Centre for Eye Research Australia, Royal \\ Victorian Eye and Ear Hospital, Melbourne, Australia and ${ }^{3}$ Department of Surgery, University of Melbourne, Melbourne, Australia
}

\begin{abstract}
The primary aim of the Guangzhou Twin Eye Study (GTES) is to explore the impact that genes and environmental influences have on common eye diseases. Since 2006, approximately 1300 pairs of twins, aged 7-15 years, were enrolled at baseline. Progressive phenotypes, such as cycloplegic refraction, axial length, height and weight, have been collected annually. Nonprogressive phenotypes such as parental refraction, corneal thickness, fundus photo, intraocular pressure and DNA were collected once at baseline. We are collaborating with fellow international twin researchers and psychologists to further explore links with general medical conditions. In this article, we review the history, major findings and future research directions for the GTES.
\end{abstract}

Keywords: Twin study; gene; environment; myopia; prediction

(Received 10 July 2019; accepted 26 October 2019)

\section{History of the Guangzhou Twin Eye Study}

The Guangzhou Twin Registry is a population-based registry of twins in Guangzhou, China. In early 2005, with the assistance of the Guangzhou City Bureau of Statistics, those who shared the same birthday, home address and household owner were selected from the Official Resident Registry as possible twins. After doublechecking the address and parents' names, a total of 9709 pairs of twins born between 1987 and 2000 were identified. Door-todoor visits were undertaken by trained interviewers from the Guangzhou City Bureau of Statistics between January and March 2006.

Following this, all twins aged 7-15 years, living in two neighboring districts of the Zhongshan Ophthalmic Center, were invited to attend an annual eye examination. A total of 705 pairs of eligible twins were invited, and 559 were examined in 2006. The sample size was further expanded by enrolling twins from other districts in Guangzhou. These additional recruitment phases to increase the sample size took place in 2008, 2009, 2010 and 2012. At present, there are 1291 sets of twins enrolled, and 11 year's follow-up visits were completed in 2018 (Table 1). Biological parents of these twins were also invited to attend the baseline examinations.

The major aim of this young twin study is to explore the etiology of common eye diseases, particularly myopia and glaucoma. Ocular phenotypes related to myopia progression, such as cycloplegic refraction, axial length (AL), height and weight were collected annually. Phenotypes not related to myopia, such as parental refraction, central corneal thickness, fundus appearance and intraocular pressure, were only collected at baseline. The systemic and ocular data collected during the study are summarized in Table 2.

Author for correspondence: Mingguang He, Email: mingguang_he@yahoo.com Cite this article: Ding X, Wang W, Scheetz J, and He M. (2019) The Guangzhou Twin Eye Study: 2019 Update. Twin Research and Human Genetics 22: 492-498, https://doi.org/ 10.1017/thg.2019.118
Approximately $10 \mathrm{ml}$ of blood was taken from all the twins at their baseline visit for DNA isolation and zygosity testing if required. All DNA were isolated by Fuji Film Quick Gene kit and stored in a $-80^{\circ} \mathrm{C}$ refrigerator. Zygosity of all same-sex twin-pairs was determined by conducting 16 multiplex short tandem repeats (PowerPlex 16 System; Promega, Madison, WI, USA) at the Forensic Medicine Department of Sun Yat-sen University. Opposite-sex twin-pairs were deemed dizygotic (DZ); therefore, they did not require genotyping.

This study was conducted in accordance with the tenets of the World Medical Association's Declaration of Helsinki. Ethical approval was obtained from the Ethics Committee of Zhongshan Ophthalmic Center. Prior to examination, written informed consent was obtained from parents or guardians of all the twins after careful explanation of the project objectives and examination procedures. This included the risks and benefits of participation, and the need to analyze DNA information for further etiology exploration.

\section{Major Findings of the GTES}

\section{Phenotypic Heritability Study and New Statistical Methods}

A principle goal of twin studies is to estimate heritability, as well as the proportion of individual phenotypic variation that can be explained by genetic variations among individuals at a given time and in a given population. Phenotypic variance encompasses several genetic effects: additive (A) or dominant (D) genetic variance; and environmental effects: shared (C) or unique (E) environmental variance. The $\mathrm{E}$ component also contains measurement error. In the design of classic twin studies, as the $\mathrm{C}$ and $\mathrm{D}$ components confound each other when pairs of twins are reared together, only one parameter (either $\mathrm{C}$ or $\mathrm{D}$ ) is allowed to be included in a single model. If the pairwise correlation in dizygotic twins is less than half of that in monozygotic (MZ) twins, it suggests that genetic 
Table 1. Sample size enrolled in each year in Guangzhou Twin Eye Study

\begin{tabular}{cccccccccc} 
& \multicolumn{7}{c}{ New enrolled } & & \multicolumn{5}{c}{ Follow-up visit } & & & \\
Year & MZ pairs & DZ pairs & Triplet & & MZ pairs & DZ pairs & Triplet & Age (Mean \pm SD) & Gender (male:female) \\
\hline 2006 & 358 & 201 & 3 & & - & - & - & $11.4 \pm 2.6$ & $1: 1.03$ \\
\hline 2007 & 1 & 1 & 1 & 310 & 166 & 3 & $12.3 \pm 2.6$ & $1: 1.05$ \\
\hline 2008 & 135 & 74 & 3 & 308 & 171 & 3 & $12.8 \pm 2.8$ & $1: 1.04$ \\
\hline 2009 & 283 & 156 & 0 & 401 & 230 & 5 & $12.6 \pm 3.2$ & $1: 1.06$ \\
\hline 2010 & 24 & 22 & 1 & 591 & 333 & 5 & $13.7 \pm 3.1$ & $1: 1.05$ \\
\hline 2011 & - & - & - & 507 & 298 & 5 & $14.4 \pm 3.0$ & $1: 1.08$ \\
\hline 2012 & 18 & 10 & 0 & 446 & 279 & 6 & $15 \pm 3.1$ & $1: 1.09$ \\
\hline 2013 & - & - & - & 465 & 291 & 3 & $16.0 \pm 3.1$ & $1: 1.07$ \\
\hline 2014 & - & - & - & 461 & 281 & 3 & $17.0 \pm 3.2$ & $1: 1.10$ \\
\hline 2015 & - & - & - & 462 & 277 & 4 & $18.2 \pm 3.2$ & $1: 1.13$ \\
\hline 2016 & - & - & - & 319 & 201 & 3 & $18.0 \pm 2.9$ & $1: 1.20$ \\
\hline 2017 & - & - & - & 313 & 287 & 4 & $19.6 \pm 3.1$ & $1: 1.13$ \\
\hline 2018 & - & - & - & 290 & 183 & 3 & $20.4 \pm 3.2$ & $1: 1.19$ \\
\hline
\end{tabular}

Note: $\mathrm{MZ}=$ monozygotic, $\mathrm{DZ}=$ dizygotic.

Table 2. Data collection in the Guangzhou Twin Study

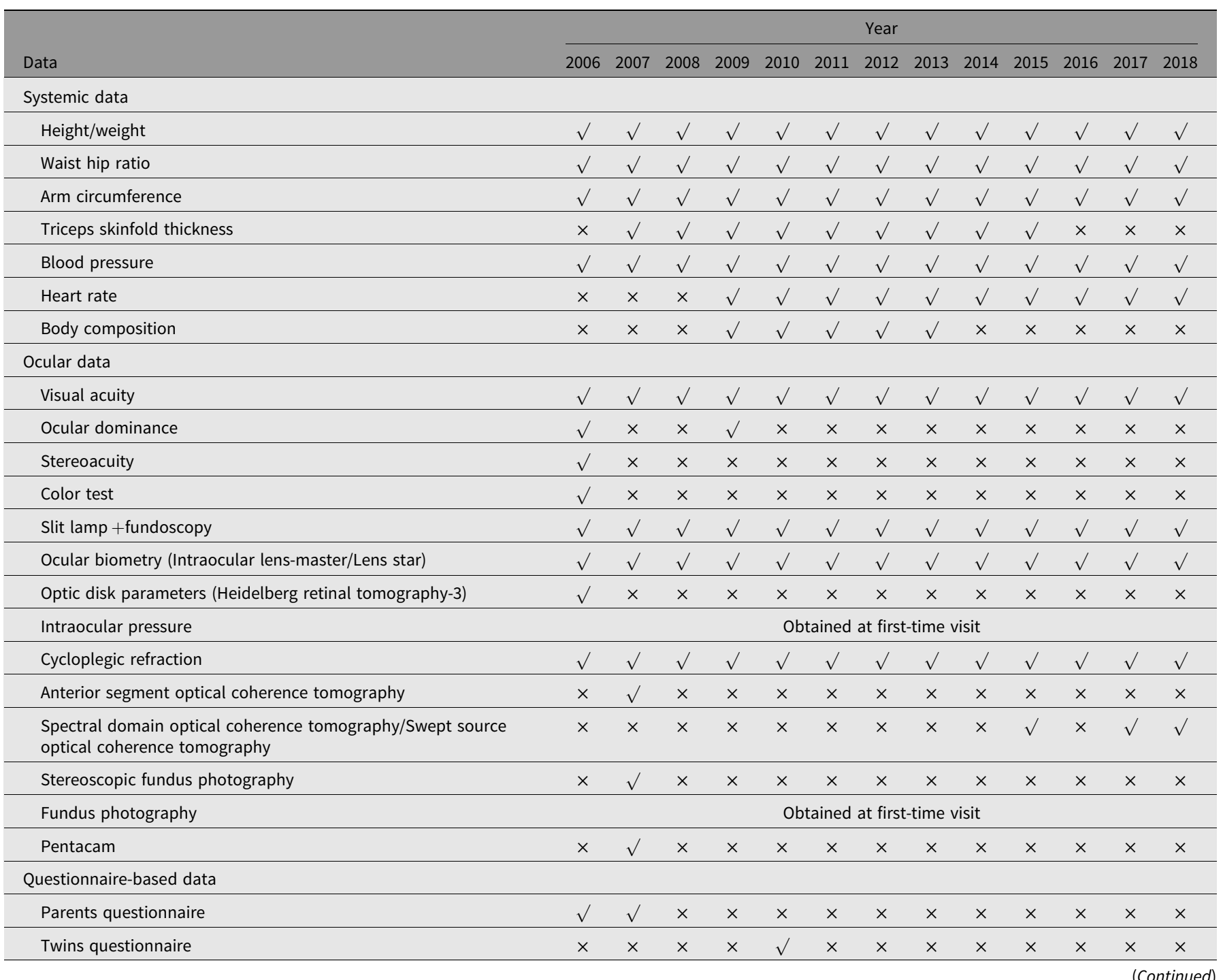


Table 2. (Continued)

\begin{tabular}{|c|c|c|c|c|c|c|c|c|c|c|c|c|c|}
\hline Data & \multicolumn{13}{|c|}{ Year } \\
\hline Balloon Analogue Risk Task test & $x$ & $x$ & $x$ & $\times$ & $\sqrt{ }$ & $x$ & $\times$ & $x$ & $x$ & $x$ & $\times$ & $x$ & $\times$ \\
\hline Conformity test & $x$ & $x$ & $x$ & $x$ & $\sqrt{ }$ & $x$ & $x$ & $x$ & $\times$ & $\times$ & $x$ & $x$ & $x$ \\
\hline \multicolumn{14}{|l|}{ Blood data } \\
\hline Glycated hemoglobin & $\times$ & $\times$ & $\times$ & $\sqrt{ }$ & $\sqrt{ }$ & $\sqrt{ }$ & $\sqrt{ }$ & $\sqrt{ }$ & $x$ & $\times$ & $\times$ & $x$ & $\times$ \\
\hline Serum lipid levels & $\times$ & $\times$ & $\times$ & $\sqrt{ }$ & $\sqrt{ }$ & $\sqrt{ }$ & $\sqrt{ }$ & $\sqrt{ }$ & $\times$ & $x$ & $\times$ & $\times$ & $\times$ \\
\hline Blood for DNA isolation & \multicolumn{13}{|c|}{ Obtained at first time visit } \\
\hline \multicolumn{14}{|l|}{ Others } \\
\hline Blood and urine tests & $\times$ & $\times$ & $x$ & $\times$ & $x$ & $\times$ & $\times$ & $\times$ & $x$ & $x$ & $\times$ & $x$ & $\sqrt{ }$ \\
\hline
\end{tabular}

Note: Collected demographic data, zygosity, birth weight, gestational age, parental lifestyles, parental education level, parental economic status, ocular and medical history of parents and twins, indoor and outdoor activities, near works.

IOL, Intraocular lens; HRT-3, Heidelberg Retinal Tomography-3; AS-OCT, anterior segment optical coherence tomography; SS-OCT, Swept Source optical coherence tomography; SD-OCT, spectral domain optical coherence tomography; IOP, intraocular pressure.

dominance is a major contributor. In this case, the saturated model is fitted with an ADE model; otherwise, the saturated model is fitted with an ACE model. Using this traditional theory, we are able to estimate myopic ocular traits such as refraction, AL, central corneal thickness, intraocular pressure, optic disk parameters, peripheral refraction and peripheral eye length, which were similarly high, ranging from 0.6 to 0.9 (Table 3) (Ding et al., 2012, 2018; He, Ge et al., 2008; He, Liu et al., 2008; He et al., 2009; Shen et al., 2012; Zheng, Ge et al., 2008; Zheng, Huang et al., 2008; Zheng et al., 2009). Our results concur with previous heritability data from the Australia (Dirani et al., 2006) and the UK twin registry (Lopes et al., 2009), which suggests there is consistency in the level of heritability across different ethnic groups and environments.

Nonetheless, it has been argued that ACE and ADE models can overestimate the phenotypic heritability, as described above. Adding parental phenotypic information into the model (extended twin family design study) allows us to distinguish the effects of $\mathrm{C}$ and D from each other (Neale \& Cardon, 1992). This shows that the ACDE model is less biased when estimating heritability than the ADE or ACE model (Keller \& Coventry, 2005). However, if we use this ACDE model when estimating the phenotypical heritability of myopia, a significant difference may exist between parents and children due to changes in environmental exposure in China over the last three decades.

We can assume there is a heterogeneous environmental effect between two generations. Therefore, we built a statistical model to test whether this heterogeneity $(\mathrm{H})$ effect existed in our myopiarelated phenotypes, named as the ACDE-H model (Guo et al., 2013). Three myopia-related biometrics - spherical equivalence (SE), AL and corneal curvature (CC) - were evaluated using three models: ACE or ADE model, using only twins' information; traditional ACDE model, using parents and twins' data together; and our ACDE-H model, using the same data as the ACDE model. We found that compared to the classic twin study (ACE model), the extended twin study (ACDE model) significantly decreases the phenotypic heritability of AL and SE, but not CC. Furthermore, differences in environmental exposures between parents and children were only significant for SE and AL, which explained about $9.6 \%$ of the variation for SE, and about $17.1 \%$ of AL variation (Ding et al., 2013). This heterogeneity effect of SE and $\mathrm{AL}$ also supports the idea that environmental change has been a significant contributor to the myopia boom in recent decades (Dolgin, 2015).

\section{Shared Genetic Determinants of Ocular and Systemic Phenotypes}

Many genetically related systemic traits coexist with ocular traits, suggesting that these phenotypes or diseases may have shared genetic pathways. In the past 12 years, a broad range of phenotypes have been collected through the GTES, which has allowed us to investigate whether correlations between phenotypes result from shared genetic factors. Using the Cholesky model, we quantified the shared genetic effects among angle opening distance (AOD), ACD and AL. We found that $23 \%$ of genetic factors are shared between AOD and ACD, 13\% between AOD and AL, and 25\% between ACD and AL (He, Hur et al., 2008). This finding confirms the strong, shared additive genetic effect of traits related to angle closure and myopia, and reveals that the pleiotropic actions of genes probably contribute to the associations between angle closure and myopia-related traits. Moreover, a significantly positive relationship was found between $\mathrm{AL}$ and height, with $89 \%$ of this phenotypic correlation due to shared genetic factors (Wang et al., 2011; Zhang et al., 2011). In addition, we have identified a significant association between cardiovascular risk factors (such as blood pressure and BMI) and retinal vascular caliber (an early marker of microvascular damage) and, more importantly, the shared genetic components for these phenotypic correlations (Xiao et al., 2015; Zheng et al., 2013). For example, $83.3 \%$ of the phenotypic correlation between mean arterial pressure and retinal arteriolar caliber was attributable to shared genetic factors, while 
Table 3. Heritability estimates using cross-sectional data from the Guangzhou Twin Study

\begin{tabular}{|c|c|c|c|c|c|c|}
\hline \multirow[b]{2}{*}{ Phenotype } & \multicolumn{2}{|c|}{$N$ twin-pairs } & \multicolumn{2}{|c|}{ Mean $\pm S D$} & \multirow{2}{*}{$\begin{array}{c}\text { Best-fitting } \\
\text { model }\end{array}$} & \multirow{2}{*}{$\begin{array}{l}\text { Heritability } \\
(95 \% \mathrm{CI})\end{array}$} \\
\hline & $\mathrm{MZ}$ & DZ & $M Z$ & DZ & & \\
\hline Spherical equivalent & 548 & 343 & $-1.75 \pm 2.52 \mathrm{D}$ & $-1.45 \pm 2.51 \mathrm{D}$ & $\mathrm{AE}$ & $0.83(0.81,0.85)$ \\
\hline Axial length & 548 & 343 & $24.2 \pm 1.25 \mathrm{~mm}$ & $24.1 \pm 1.22 \mathrm{~mm}$ & ACE & $0.66(0.53,0.81)$ \\
\hline Corneal curvature & 548 & 343 & $43.5 \pm 1.42 \mathrm{D}$ & $43.4 \pm 1.49 \mathrm{D}$ & $\mathrm{AE}$ & $0.89(0.87,0.90)$ \\
\hline \multicolumn{7}{|l|}{ Peripheral axial length } \\
\hline PEL-T40 & 104 & 54 & $22.96 \pm 0.95 \mathrm{~mm}$ & $22.97 \pm 0.86 \mathrm{~mm}$ & $\mathrm{AE}$ & $0.86(0.81,0.90)$ \\
\hline PEL-N40 & 104 & 54 & $23.01 \pm 1.04 \mathrm{~mm}$ & $23.05 \pm 0.91 \mathrm{~mm}$ & $\mathrm{AE}$ & $0.90(0.86,0.93)$ \\
\hline RPEL-T40 & 104 & 54 & $-1.30 \pm 0.58 \mathrm{~mm}$ & $-1.28 \pm 0.58 \mathrm{~mm}$ & $\mathrm{AE}$ & $0.80(0.72,0.85)$ \\
\hline RPEL-N40 & 104 & 54 & $-1.25 \pm 0.53 \mathrm{~mm}$ & $-1.20 \pm 0.66 \mathrm{~mm}$ & $\mathrm{AE}$ & $0.76(0.67,0.82)$ \\
\hline \multicolumn{7}{|l|}{ Peripheral refraction } \\
\hline PR-T40 & 72 & 48 & $-0.50 \pm 2.10 \mathrm{D}$ & $0.03 \pm 1.89 \mathrm{D}$ & $\mathrm{AE}$ & $0.84(0.77,0.90)$ \\
\hline PR-N40 & 72 & 48 & $0.29 \pm 2.38 \mathrm{D}$ & $0.81 \pm 1.81 \mathrm{D}$ & $\mathrm{AE}$ & $0.76(0.66,0.84)$ \\
\hline RPRE-T40 & 72 & 48 & $0.91 \pm 1.33 \mathrm{D}$ & $1.24 \pm 1.39 \mathrm{D}$ & $\mathrm{AE}$ & $0.63(0.47,0.74)$ \\
\hline RPRE-N40 & 72 & 48 & $1.70 \pm 1.90 \mathrm{D}$ & $2.02 \pm 1.49 \mathrm{D}$ & $\mathrm{AE}$ & $0.70(0.58,0.79)$ \\
\hline T40-N40 Asymmetry & 72 & 48 & $-0.79 \pm 1.65 \mathrm{D}$ & $-0.79 \pm 1.36 \mathrm{D}$ & $\mathrm{AE}$ & $0.55(0.39,0.68)$ \\
\hline Central corneal thickness & 297 & 152 & $551.9 \pm 34.0 \mu \mathrm{m}$ & $551.7 \pm 30.3 \mu \mathrm{m}$ & $\mathrm{AE}$ & $0.91(0.89,0.93)$ \\
\hline Anterior chamber depth & 357 & 206 & $3.5 \pm 0.3 \mathrm{~mm}$ & $3.5 \pm 0.3 \mathrm{~mm}$ & $\mathrm{AE}$ & $0.90(0.82,0.92)$ \\
\hline Lens thickness & 482 & 286 & $3.5 \pm 0.2 \mathrm{~mm}$ & $3.5 \pm 0.2 \mathrm{~mm}$ & $\mathrm{AE}$ & $0.90(0.88,0.91)$ \\
\hline Angle opening distance at $500 \mu \mathrm{m}$ & 305 & 157 & $0.66 \pm 0.24 \mathrm{~mm}$ & $0.68 \pm 0.22 \mathrm{~mm}$ & $\mathrm{AE}$ & $0.70(0.64,0.75)$ \\
\hline Anterior recess area & 305 & 157 & $0.53 \pm 0.21 \mathrm{~mm}^{2}$ & $0.55 \pm 0.18 \mathrm{~mm}^{2}$ & $\mathrm{AE}$ & $0.73(0.68,0.78)$ \\
\hline Trabecular-iris space area at $750 \mu \mathrm{m}$ & 305 & 157 & $0.44 \pm 0.15 \mathrm{~mm}^{2}$ & $0.46 \pm 0.14 \mathrm{~mm}^{2}$ & $\mathrm{AE}$ & $0.71(0.65,0.76)$ \\
\hline Iris thickness & 309 & 165 & $0.4 \pm 0.6 \mathrm{~mm}$ & $0.4 \pm 0.1 \mathrm{~mm}$ & $\mathrm{AE}$ & $0.59(0.51,0.65)$ \\
\hline Pupil size & 309 & 165 & $5.6 \pm 0.8 \mathrm{~mm}$ & $5.6 \pm 0.9 \mathrm{~mm}$ & $\mathrm{AE}$ & $0.63(0.57,0.69)$ \\
\hline Optic disk area & 355 & 202 & $2.0 \pm 0.4 \mathrm{~mm}$ & $2.0 \pm 0.5 \mathrm{~mm}$ & $\mathrm{AE}$ & $0.77(0.73,0.81)$ \\
\hline Cup area & 355 & 202 & $0.5 \pm 0.5 \mathrm{~mm}$ & $0.5 \pm 0.3 \mathrm{~mm}$ & $\mathrm{AE}$ & $0.83(0.79,0.86)$ \\
\hline Cup-to-disk ratio & 355 & 202 & $0.2 \pm 0.1$ & $0.3 \pm 0.1$ & $\mathrm{AE}$ & $0.78(0.75,0.82)$ \\
\hline Intraocular pressure & 309 & 164 & $14.2 \pm 2.3 \mathrm{mmHg}$ & $14.2 \pm 2.2 \mathrm{mmHg}$ & $\mathrm{AE}$ & $0.67(0.61,0.72)$ \\
\hline
\end{tabular}

$A=$ additive genetic effect; $C=$ common environmental effect; $E=$ unique environmental effect; $D=$ diopter; $P R=$ peripheral refraction; $R P R E=$ relative peripheral refractive error; $\mathrm{PEL}=$ peripheral eye length; $\mathrm{RPEL}=$ relative peripheral eye length; $\mathrm{T} 40=$ temporal $40^{\circ} ; \mathrm{N} 40=$ nasal $40^{\circ}$.

the phenotypic correlation between BMI and retinal arteriolar caliber was nearly $100 \%$. These genetic findings indicate the important influence that shared genetic factors have on ocular and systemic diseases. There is also the potential to identify genes unique to each phenotype, which may help to unravel the underlying pathogenesis of its correlated phenotypes.

\section{Environmental Factor Estimation on Myopia}

School-age myopia is a complex eye condition. It involves genes, the environment and gene-environment interactions. Near work and outdoor activity are considered two important environmental factors. However, previous environmental estimations cannot adjust for the genetic background from environmental exposure or be adjusted by including parental refraction in the regression model, which is a relatively crude and contestable method. MZ twins share age, gender, genetic background and family culture. So, it is reasonable to assume that differences in environmental exposures are responsible for the discordance among $\mathrm{MZ}$ twins.

We used this MZ twin control methodology to explore the effect of near work and outdoor time on myopia. A standard questionnaire was used to collect data on near work and time spent outdoors (He et al., 2015). Using mixed-model analysis, we found that difference in the amount of near work was a risk factor for discordance in myopic SE. Furthermore, the interaction between time spent outdoors and age was a protective factor for discordance in myopic SE, but the overall association between difference in the amount of time spent outdoors and SE discordance was not significant. Furthermore, the difference in near work and time spent outdoors explained only about $1.8 \%$ and $2.5 \%$ of the variation in SE discordance, respectively.

\section{Collaborative Findings with Domestic and Overseas Researchers}

In 2013, we received an invitation to COllaborate with the Development of Anthropometrical measures in Twins (CODA Twins) consortium, which aims to enroll a large sample of twins from birth to old age to explore the effects of genetic and environmental factors on height, weight and BMI (Jelenkovic et al., 2015; Silventoinen et al., 2015, 2016). We also collaborated with psychologists to explore factors influencing risk-taking propensity and 
intellectual quotient (IQ) of twins using the Balloon Analogue Risk Task and the Wechsler Intelligence Scale (WISC-IV-Chinese version). The multivariate regression model revealed that risk-taking propensity increased significantly with increasing age. Furthermore, higher IQ was significantly associated with lower SNP. Our team developed two behavior tests - word memorization and price estimation - to examine the conformity of twins. We found that social conformity was inheritable, with a heritability estimation of 0.25 to 0.37 , providing a basis for further exploration of the molecular mechanism of conformity. In addition, we identified several inherited susceptibility genes related to social conformity, including NAV3, PTPRD, ARL10 and CTNND2 using genomewide association study analysis (Chen, Zhu et al., 2018).

\section{Current Works and Future Plan}

The previous focus of the GTES was to establish a longitudinal cohort of ocular disease of twins from childhood to adulthood. The available 13 year's annual data are particularly valuable for identifying genetic factors and the effects of early environmental exposure on ocular and systemic diseases. After completing the 13 year's follow-up, most participants had entered adulthood. Therefore, one of our current efforts is to analyze longitudinal data to quantify the longitudinal phenotype changes and potential predictors. In addition, we plan to extend our study to the following areas to maximize the scientific potential of the project:

1. Quantitative analysis of longitudinal phenotype changes and influence of genetic and environmental factors. The initial goals of the study were to identify and quantify the genetic components that account for the variation in common ocular phenotypes such as SE and AL, and these have been fulfilled. However, the longitudinal change of ocular phenotypes in twins is unclear. In addition, the genetic effects of many novel phenotypes, including in-vivo imaging metrics, were obtained by advanced imaging devices (such as vessel density and foveal avascular zone from optical coherence tomography angiography) and new molecular biomarkers in blood and urine (such as levels of noncoding RNA expression, methylation levels at $\mathrm{CpG}$ sites and serum metabolite). Finally, we are working on the quantification of dynamic heritability in this longitudinal cohort.

2. Identifying the key structural and functional differences from full-body multimodality imaging between $M Z$ twins. Each individual is unique, including identical MZ twins (Brodin et al., 2015; van Dongen et al., 2012). Researchers are keen to understand intratwin differences and the mechanism of monozygosity. Various studies have identified the structural difference between MZ twins; for example, morphological analysis of three-dimensional magnetic resonance imaging (MRI) revealed that brain volume measures were highly correlated within MZ twins but surface measures of the brain were influenced by environmental factors (de Manzano \& Ullen, 2018; Oppenheim et al., 1989; Steinmetz et al., 1994; White et al., 2002). However, whether the key differences resulted from genetic factors, environmental factors or at random are poorly understood due to the limitations of the imaging modality. With the development of more sophisticated in-vivo devices, total body imaging will be able to better characterize the structure and molecular processes in humans. Among these, the combined positron emission tomography (PET) and MRI imaging enables simultaneous imaging, providing molecular, morphological and functional information (Cherry et al., 2018; Sauter et al., 2010). Multimodality imaging data of a subgroup of 20 pairs of MZ twins from the GTES were acquired using total body PET/MRI scanning, chest computed tomography (CT) scanning, echocardiography, vascular ultrasound and ocular imaging. This subgroup of MZ twins also underwent wealth assays, including regular blood and urine tests, serum electrolyte, complete lipid and metabolic panels, serum viscosity, immune system biomarkers, metabolic system, endocrinal system, kidney and liver. In addition, participants completed various surveys in relation to medical history, diet, physical activity, sleep, cognition and stress. Wearable devices were also equipped to obtain information on activity levels, sleep, physiology and continuous glucose monitoring. Indepth analysis is ongoing to detect key differences in image-derived phenotypes within MZ twins, which will pave the way for a better understanding of the biological difference of MZ twins.

3. Continuous collection of multidimensional, dense, dynamic data clouds of MZ twins from a longitudinal big data approach. The precision medicine (personalized, P4 or stratified medicine) outcomes highlighted the medical decision based on personal characteristics, including molecular and behavioral biomarkers, rather than the average level of population. The recent advances in multiomics technology and wearable devices have enabled deep molecular analysis and physiological monitoring, providing a vital source of big data for precision medicine (Chen et al., 2012; Chen, Xia et al., 2018; Schussler-Fiorenza Rose et al., 2019). Precision medicine depends on data science, especially machine learning (Karczewski \& Snyder, 2018; Trachana et al., 2018). There is enthusiasm for the potential of big data and machine learning for precision medicine and health, but few examples are currently available in the literature. MZ twins provide a unique opportunity for practicing precision medicine because they are perfectly matched in terms of age, gender, genome and early-life environmental factors.

We plan to collect personal, multidimensional, dense, dynamic data clouds of MZ twins using a longitudinal big data approach. Participants will regularly receive multiomic tests, physiological examinations, total body and ocular imaging, and will be continuously monitored using wearable devices. Biological samples such as blood, saliva, urine and stool will be collected for obtaining multi-omic data, including DNA methylation, transcriptome, metabolome, proteome, lipidome, antibodome and microbiome. Continuous monitoring using wearable sensors is ongoing, collecting the lifestyle and physiological information for near work, physical activity, sleep, body composition and heart rate, as well as environmental information such as temperature, rainfall, humidity, wind speed, particulate matter and radiation exposure. In addition, various phone applications are applied to allow participants to easily report changes in health and lifestyle, such as diet, exercise, stress, mood and disease. The integrated cloud data enable deep profiling to create a huge 'personal biology map' for each MZ pair. We are currently seeking multidisciplinary collaborators for analysis of the deep personal dataset using big data and machine learning technologies with the aim to (1) identify the key differences within $\mathrm{MZ}$ twins resulting from nongenetic influences by exploring deep longitudinal profiling; (2) discover novel 
molecular biomarkers, behavior or environmental factors that may impact human health; and (3) develop prediction models for medical conditions using integrated measurements. GTES data collection is still ongoing and will last a lifetime. We are open to new collaborations. Any requests for cooperation or biobank data sharing can be made by contacting Professor He Mingguang (mingguang_he@yahoo.com).

Financial support. The research funded by National Natural Science Foundation of China (81600763) and Fundamental Research Funds of the State Key Laboratory. Prof. Mingguang receives support from the University of Melbourne Research Accelerator Program and the CERA Foundation. The Centre for Eye Research Australia receives Operational Infrastructure Support from the Victorian State Government. The sponsor or funding organization had no role in the design or conduct of this research.

Conflict of interest. The authors have no financial or other conflicts of interest concerning this study.

\section{References}

Brodin, P., Jojic, V., Gao, T., Bhattacharya, S., Angel, C. J., Furman, D., . . . Davis, M. M. (2015). Variation in the human immune system is largely driven by non-heritable influences. Cell, 160, 37-47.

Chen, B., Zhu, Z., Wang, Y., Ding, X., Guo, X., He, M., . . Rao, Y. (2018). Nature vs. nurture in human sociality: Multi-level genomic analyses of social conformity. Journal of Human Genetics, 63, 605-619.

Chen, R., Mias, G. I., Li-Pook-Than, J., Jiang, L., Lam, H. Y., Miriami, E., . . Snyder, M. (2012). Personal omics profiling reveals dynamic molecular and medical phenotypes. Cell, 148, 1293-1307.

Chen, R., Xia, L., Tu, K., Duan, M., Kukurba, K., Li-Pook-Than, J., Xie, D., \& Snyder, M., (2018). Longitudinal personal DNA methylome dynamics in a human with a chronic condition. Nature Medicine, 24, 1930-1939.

Cherry, S. R., Jones, T., Karp, J. S., Qi, J., Moses, W. W., \& Badawi, R. D. (2018). Total-body PET: Maximizing sensitivity to create new opportunities for clinical research and patient care. Journal of Nuclear Medicine, 59, 3-12.

de Manzano, O., \& Ullen, F. (2018). Same genes, different brains: Neuroanatomical differences between monozygotic twins discordant for musical training. Cerebral Cortex, 28, 387-394.

Ding, X., Chen, Y., \& He, M. (2018). An update of the Guangzhou Twin Eye Study. Annals of Eye Science, 3, 38-38.

Ding, X., Guo, X., Morgan, I.G., \& He, M. (2013, May). Heterogeneous environmental effect on myopia in parents and their children — The Guangzhou Twin Eye Study. Paper presented at ARVO Annual Meeting, Seattle, WA.

Ding, X., Lin, Z., Huang, Q., Zheng, Y., Congdon, N., \& He, M. (2012). Heritability of peripheral refraction in Chinese children and adolescents: The Guangzhou Twin Eye Study. Investigative Ophthalmology \& Visual Science, 53, 107-111.

Dirani, M., Chamberlain, M., Shekar, S. N., Islam, A. F., Garoufalis, P., Chen, C. Y., . . Baird, P. N. (2006). Heritability of refractive error and ocular biometrics: The Genes in Myopia (GEM) twin study. Investigative Ophthalmology \& Visual Science, 47, 4756-4761.

Dolgin, E. (2015). The myopia boom. Nature, 519, 276-278.

Guo, X., Liu, D., Wen, C., He, M., \& Wang, X. (2013). Incorporating heterogeneous parent-child environmental effects in biometrical genetic models. Statistics in Medicine, 32, 3501-3508.

He, M., Ge, J., Wang, D., Zhang, J., Hewitt, A. W., Hur, Y. M., . . F Foster, P. J. (2008). Heritability of the iridotrabecular angle width measured by optical coherence tomography in Chinese children: The Guangzhou Twin Eye Study. Investigative Ophthalmology \& Visual Science, 49, 1356-1361.

He, M., Hur, Y. M., Zhang, J., Ding, X., Huang, W., \& Wang, D. (2008). Shared genetic determinant of axial length, anterior chamber depth, and angle opening distance: The Guangzhou Twin Eye Study. Investigative Ophthalmology \& Visual Science, 49, 4790-4794.

He, M., Liu, B., Huang, W., Zhang, J., Yin, Q., Zheng, Y., .. . Ge, J. (2008). Heritability of optic disc and cup measured by the Heidelberg Retinal
Tomography in Chinese: The Guangzhou Twin Eye Study. Investigative Ophthalmology \& Visual Science, 49, 1350-1355.

He, M., Wang, D., Console, J.W., Zhang, J., Zheng, Y., \& Huang, W. (2009). Distribution and heritability of iris thickness and pupil size in Chinese: The Guangzhou Twin Eye Study. Investigative Ophthalmology \& Visual Science, 50, 1593-1597.

He, M., Xiang, F., Zeng, Y., Mai, J., Chen, Q., Zhang, J., .. Morgan, I. G. (2015). Effect of time spent outdoors at school on the development of myopia among children in China: A randomized clinical trial. JAMA, 314, 1142-1148.

Jelenkovic, A., Yokoyama, Y., Sund, R., Honda, C., Bogl, L. H., Aaltonen, S., .. Silventoinen, K. (2015). Zygosity differences in height and body mass index of twins from infancy to old age: A study of the CODATwins Project. Twin Research and Human Genetics, 18, 557-570.

Karczewski, K. J., \& Snyder, M. P. (2018). Integrative omics for health and disease. Nature Reviews Genetics, 19, 299-310.

Keller, M. C., \& Coventry, W. L. (2005). Quantifying and addressing parameter indeterminacy in the classical twin design. Twin Research and Human Genetics, 8, 201-213.

Lopes, M. C., Andrew, T., Carbonaro, F., Spector, T. D., \& Hammond, C. J. (2009). Estimating heritability and shared environmental effects for refractive error in twin and family studies. Investigative Ophthalmology \& Visual Science, 50, 126-131.

Neale, M., \& Cardon, L. (1992). Methodology for genetic studies of twins and families. Dordrecht, the Netherlands: Kluwer Academic Publishers BV.

Oppenheim, J. S., Skerry, J. E., Tramo, M. J., \& Gazzaniga, M. S. (1989). Magnetic resonance imaging morphology of the corpus callosum in monozygotic twins. Annals of Neurology, 26, 100-104.

Sauter, A. W., Wehrl, H. F., Kolb, A., Judenhofer, M. S., \& Pichler, B. J. (2010). Combined PET/MRI: One step further in multimodality imaging. Trends in Molecular Medicine, 16, 508-515.

Schussler-Fiorenza, R. S. M., Contrepois, K., Moneghetti, K. J., Zhou, W., Mishra, T., Mataraso, S., ... Snyder, M. P. (2019). Longitudinal big data approach for precision health. Nature Medicine, 25, 792-804.

Shen, P., Ding, X., Zheng, Y., Congdon, N. G., \& He, M. (2012). Contribution of genetic and environmental effects on lens thickness: The Guangzhou Twin Eye study. Investigative Ophthalmology \& Visual Science, 53, 1758-1763.

Silventoinen, K., Jelenkovic, A., Sund, R., Honda, C., Aaltonen, S., Yokoyama, Y., . . Kaprio, J. (2015). The CODATwins Project: The cohort description of Collaborative Project of Development of Anthropometrical Measures in Twins to Study Macro-Environmental Variation in Genetic and Environmental Effects on Anthropometric Traits. Twin Research and Human Genetics, 18, 348-360.

Silventoinen, K., Jelenkovic, A., Sund, R., Hur, Y. M., Yokoyama, Y., Honda, C., ... Kaprio, J. (2016). Genetic and environmental effects on body mass index from infancy to the onset of adulthood: An individual-based pooled analysis of 45 twin cohorts participating in the COllaborative project of Development of Anthropometrical measures in Twins (CODATwins) study. The American Journal of Clinical Nutrition, 104, 371-379.

Steinmetz, H., Herzog, A., Huang, Y., \& Hacklander, T. (1994). Discordant brain-surface anatomy in monozygotic twins. The New England Journal of Medicine, 331, 952-953.

Trachana, K., Bargaje, R., Glusman, G., Price, N. D., Huang, S., \& Hood, L. E. (2018). Taking Systems Medicine to Heart. Circulation Research, 122, 1276-1289.

van Dongen, J., Slagboom, P. E., Draisma, H. H., Martin, N. G., \& Boomsma, D. I. (2012). The continuing value of twin studies in the omics era. Nature Reviews Genetics, 13, 640-653.

Wang, D., Ding, X., Liu, B., Zhang, J., \& He, M. (2011). Longitudinal changes of axial length and height are associated and concomitant in children. Investigative Ophthalmology \& Visual Science, 52, 7949-7953.

White, T., Andreasen, N. C., \& Nopoulos, P. (2002). Brain volumes and surface morphology in monozygotic twins. Cerebral Cortex, 12, 486-493.

Xiao, W., Gong, W., Chen, Q., Ding, X., Chang, B., \& He, M. (2015). Association between body composition and retinal vascular caliber in children and adolescents. Investigative Ophthalmology \& Visual Science, 56, $705-710$. 
Zhang, J., Hur, Y. M., Huang, W., Ding, X., Feng, K., \& He, M. (2011). Shared genetic determinants of axial length and height in children: The Guangzhou Twin Eye Study. Archives of Ophthalmology 129, 63-68.

Zheng, Y., Ge, J., Huang, G., Zhang, J., Liu, B., Hur, Y. M., \& He, M. (2008). Heritability of central corneal thickness in Chinese: The Guangzhou Twin Eye Study. Investigative Ophthalmology \& Visual Science, 49, 4303-4307.

Zheng, Y., Huang, G., Huang, W., \& He, M. (2008). Distribution of central and peripheral corneal thickness in Chinese children and adults: The Guangzhou twin eye study. Cornea, 27, 776-781.
Zheng, Y., Huang, W., Zhang, J., \& He, M. (2013). Phenotypic and genetic correlation of blood pressure and body mass index with retinal vascular caliber in children and adolescents: The Guangzhou Twin Eye Study. Investigative Ophthalmology \& Visual Science, 54, 423-428.

Zheng, Y., Xiang, F., Huang, W., Huang, G., Yin, Q., \& He, M. (2009). Distribution and heritability of intraocular pressure in chinese children: The Guangzhou twin eye study. Investigative Ophthalmology \& Visual Science, 50, 2040-2043. 